\title{
A compact readout system for the R3B High-Resolution Neutron Time-of-Flight Spectrometer (NeuLAND) ${ }^{* \dagger}$
}

\author{
C. Ugur ${ }^{\ddagger 1}$, K. Koch ${ }^{1}$, J. Hoffmann ${ }^{1}$, M. Heil ${ }^{1}$, and the FAIR@GSI project ${ }^{1}$ \\ ${ }^{1}$ GSI, Darmstadt, Germany
}

The R3B High-Resolution Neutron Time-of-Flight Spectrometer consists of up to 30 double planes each containing 100 sub modules of plastic scintillators, which are read out on the far ends by two PMTs. For these 6000 channels a new compact and modular readout system has been developed. The electronics setup is divided into 16 channel amplifier and fast comparator boards. Digitization units using FPGA TDC technology with a timing precision of about $10 \mathrm{ps} \mathrm{rms}$ are used. Optional charge measuring boards based on the method of conversion of charge to time-over-threshold and configuration boards to parametrize thresholds, timings, etc. are also available.

The concept of the former readout electronics, TACQUILA, of the LAND experiment is taken as a base for NeuLAND. First, the TAC stage has been replaced by a high-precision TDC in an FPGA implemented on the new timing digitization board, TAMEX, which is designed as a multi-channel front-end electronics card for high precision time and charge measurements. Second, due to the higher data rate and the required enhanced precision, a new charge measuring unit, based on the method of conversion of charge to time-over-threshold, has been developed (QTC).

In addition to service boards, power boards and optical interfaces etc., the main electronics is divided into four parts. The front-end board (LANDFEE) receives the 16 incoming signals from the detector PMTs. These signals are galvanically isolated from the successional circuitry by appropriate transformers. In the next step the signal is divided into two parts: An analog part for charge measurement purposes and the second branch is directed to a fast comparator for timing determination.

The analog signal from LANDFEE is send to the charge measuring board (QTC), where the shaping and integration of the incoming signal takes part. By the method of time-over-threshold, this board delivers a logical signal directly to TAMEX. In parallel, the timing information travels through a connector to TAMEX to determine the timing of the leading and trailing edges of the signal.

An FPGA on TAMEX is used, among other things, as a fast 16 channel TDC. The power and trigger distribution to the cards is done through the backplanes and PCI-express connectors. The data transfer is also done through the back-

\footnotetext{
* This report is the summary of the conference report submitted at the "2014 IEEE Nuclear Science Symposium and Medical Imaging Conference" in 08-15 November 2015 in Seattle, WA, USA. The conference report is currently pending.

$\dagger$ Work supported by FAIR@GSI PSP 1.2.5.1.2.5.

$\ddagger$ c.ugur@gsi.de
}

planes and optical links using the Multi Branch System MBS.

In order to handle the thresholds, monitor the analog channels, generate a logic OR, build a multiplicity and other things, a dedicated control card, TRIPLEX[2], has been developed as well. With an appropriate branch structure, including the analog signals as well as the digital ones, all planes can be configured and monitored from a central place.

The electronics is tested in the laboratory with a pulser with realistic detector signal shape for the charge and time measurements. The system has charge and time precisions of $0.9 \%$ and 16 ps respectively (Figure 1 ).
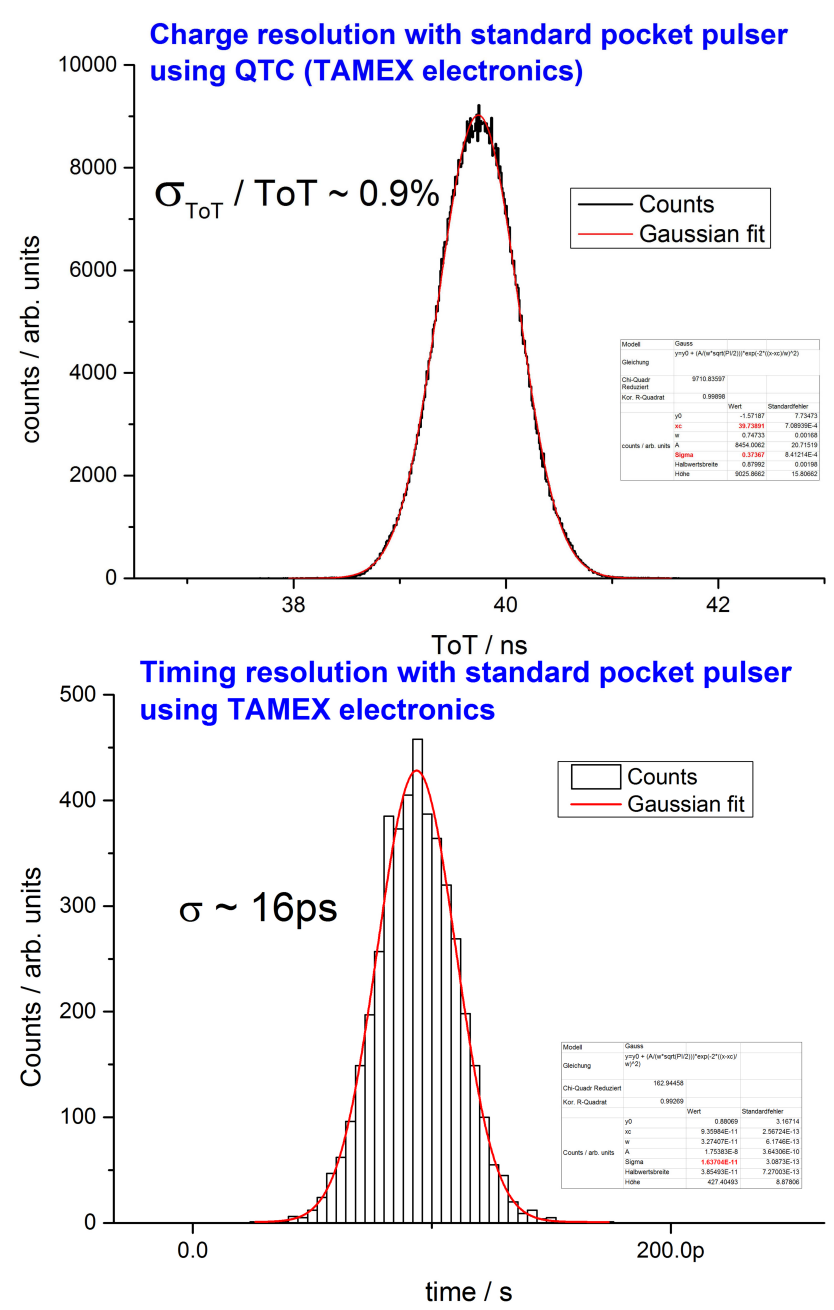

Figure 1: Charge (above) and timing (below) measurements of a pulser with realistic detector signal shape. 
The system is also tested under heavy ion beam conditions and the first test experiments performed at GSI in October 2014 show that the requirements on charge and time precision are met. The results of these test experiments are discussed by Heil et.al [1] in this GSI Scientific Report.

\section{References}

[1] M. Heil et.al, "In-beam tests of a new ToF wall for the R3B setup”, This GSI Scientific Report.

[2] K. Koch et.al, "TRIPLEX, an Upgrade for the TACQUILA System”, GSI Scientific Report 2010, PHN-IS-EE-07. 